\title{
SOLUCIÓN GLOBAL PARA UNA CLASE DE ECUACIONES ABSTRACTAS DEGENERADAS ASOCIADA A LA ECUACIÓN NO-LINEAL DE LA VIGA
}

Raúl Izaguirre Maguiña Eugenio Cabanillas Lapa ${ }^{2}$

\section{RESUMEN}

En este trabajo estudiamos la existencia y unicidad de solución débil para el problema abstracto:

$$
\begin{aligned}
& u^{\prime \prime}(t)+A^{\varepsilon} u(t)+M\left(\left|\begin{array}{ll}
A^{\alpha} u & t
\end{array}\right|^{2}\right) A^{\beta} u(t)=f(t) \\
& u(0)=u_{0} ; \quad u^{\prime}(0)=u_{1}
\end{aligned}
$$

El operador lineal $A$, está definido por la terna $\{H, V, a(\mathrm{u}, \mathrm{v})\}$, donde $H, V$, son espacios de Hilbert, la inmersión de $V$ en $H$ es densa y compacta. La forma bilineal $a$ es no-negativa, la función no - lineal $\mathrm{M}(\mathrm{s})$ es a valores reales, acotada inferiormente, es decir existe $\sigma \in \mathbf{R}$ tal que:

$$
M(s) \geq-\sigma, \forall s \geq 0, \quad \sigma \in \mathbf{R}
$$

Los exponentes, $\alpha, \beta, \varepsilon$, son números reales no negativos, cumpliendo ciertas condiciones. La ecuación (*) contiene (entre otros) la ecuación no-lineal de la viga.

\section{Introducción}

Sea $\Omega$ un abierto de $\mathrm{R}^{\mathrm{n}}$ con frontera regular; $Q$ el cilindro $\left.\Omega \times\right] 0, T[, 0<T<\infty$, con frontera lateral $\Sigma$. La siguiente ecuación diferencial parcial no-lineal.

$$
\begin{array}{ll}
u_{t t}+\Delta^{2} u+M\left(\int_{\Omega}|\nabla u|^{2} d x\right)(-\Delta u)=f & \text { en } Q \\
u=0 & \text { en } \Sigma \\
u(0)=u_{0} ; u_{t}(0)=u_{1} & \text { en } \Omega
\end{array}
$$


Es un modelo n-dimensional de la ecuación que describe las vibraciones no-lineales de una viga de extensión finita, que para dimensión $\mathrm{n}=1$ tienen la forma (ver [16]);

$$
\frac{\partial^{2} u}{\partial t^{2}}+\frac{E I \partial^{4} u}{\xi \partial x^{4}}-\left(\frac{H}{\xi}+\frac{E A}{2 \xi L} \int_{0}^{L}\left(\frac{\partial u}{\partial x}\right)^{2} d x\right) \frac{\partial^{2} u}{\partial x^{2}}=0
$$

donde $\mathrm{E}$ es el módulo de Young del material, I el momento de inercia, $\xi$ representa la densidad y A el área de la sección recta.

Asimismo la ecuación (1) desde el punto de vista de los métodos abstractos en espacios de Hilbert, puede ser considerada como un caso particular de:

$$
\begin{aligned}
& u^{\prime \prime}(t)+A^{\varepsilon} u(t)+M\left(\left|A^{\alpha} u(t)\right|^{2}\right) A^{\beta} u(t)=f(t) \\
& u(0)=u_{0} ; u^{\prime}(0)=u_{1}
\end{aligned}
$$

Donde A es un operador autoadjunto, no acotado y positivo, definido por la terna $\{H, V,(())$,$\} ,$ donde $H, V$, son espacios de Hilbert, la inmersión de $V$ en $H$ es densa y compacta; variantes de la ecuación abstracta (3), son por ejemplo:

$$
\begin{aligned}
& u^{\prime \prime}+M\left(\left|A^{1 / 2} u\right|^{2}\right) A u+A^{\alpha} u^{\prime}=f \\
& K(x, t) u^{\prime \prime}+A u^{\prime}+M\left(\left|A^{1 / 2} u\right|^{2}\right) A u=f \\
& K u^{\prime \prime}+A^{2} u+M\left(\left|A^{1 / 2} u\right|^{2}\right) A u+u^{\prime}=f
\end{aligned}
$$

La Ecuación (4), es estudiada entre otros por L. Medeiros-M. Milla [12], quienes prueban la existencia de soluciones globales para $\mathrm{M}(\mathrm{s}) \geq \mathrm{m}_{0}>0$, A un operador estrictamente positivo y $\alpha \in[0,1]$. El caso degenerado para la ecuación (4) es tratado por M. Aassila en [2] donde se obtiene existencia y unicidad de solución global considerando que $M(s) \geq 0$ y que el operador A es no-negativo, $\alpha=1$. Solución global para ecuaciones del tipo (5), son estudiadas por ejemplo en D. Pereira-R. Izaguirre [6], que obtienen solución global única, y donde la función $K(x, t) \geq 0$. En [14] Pereira D. y en [13] Muñoz J. estudian la existencia, unicidad y decaimiento de la energía para la ecuación (6).

Estudios sobre modelos en ecuaciones hiperbólicas no-lineales que incluyen el operador de Kirchhoff-Carrier son por ejemplo los presentados en [3] Cousin-Larkin-Frota-Medeiros y en [8], Izaguirre-Veliz que tratan ecuaciones de la forma:

$$
u^{\prime \prime}(t)+M\left(\left|A^{\alpha} u(t)\right|^{2}\right) A^{\beta} u(t)=f(t)
$$


donde la función no-lineal $M$ es de clase $C^{1}$ y estrictamente positiva. En [3], se obtiene soluciones globales con datos analíticos "suficientemente pequeños" . En [8], se obtiene solución local, pero en una clase mayor de datos iniciales.

El caso degenerado de la ecuación (7) es tratado en la referencia [7], donde se determina la existencia y unicidad de solución local débil.

2. Caso No Degenerado. Sean $(V,(())),,(H,()$,$) espacios de Hilbert, la inmersión de V$ en $H$ densa y compacta. Sea también $A$ el operador definido por la terna $\{V, H ;(())$,$\} .$

Entonces, $D(A)$ es un subespacio denso en $H, A$ es un operador no-acotado auto-adjunto y positivo de $H$,con espectro discreto;

$$
A w_{v}=\lambda_{v} w_{v} \quad \forall v=1,2 \ldots \ldots ; \lambda_{v} \rightarrow \infty \text { cuando } v \rightarrow \infty
$$

$\left\{w_{v}\right\}$ es un sistema ortonormal completo de $H$ de modo que:

$$
\begin{aligned}
& D(A)=\left\{u \in H ; \sum_{v=1}^{\infty} \lambda_{v}^{2}\left|\left(u, w_{v}\right)\right|^{2} \prec \infty\right\} \\
& A u=\sum_{v=1}^{\infty} \lambda_{v}\left(u, w_{v}\right) w_{v} ; \forall u \in D(A)
\end{aligned}
$$

Asimismo para todo $\alpha \in \mathbf{R}$, el operador $A^{\alpha}$ está bien definido

$$
\begin{aligned}
& D\left(A^{\alpha}\right)=\left\{u \in H ; \sum_{v=1}^{\infty} \lambda_{v}^{2 \alpha} \mid\left(u, w_{v}\right)^{2} \prec \infty\right\} \\
& A^{\alpha} u=\sum_{v=1}^{\infty} \lambda_{v}^{\alpha}\left(u, w_{v}\right) w_{v} ; \forall u \in D(A)
\end{aligned}
$$

Definiendo:

$$
|u|_{D(\alpha)}^{2}=|u|_{\alpha}^{2}=\sum_{v=1}^{\infty} \lambda_{v}^{2 \alpha}\left|\left(u, w_{v}\right)\right|^{2} ; \quad u \in D\left(A^{\alpha}\right)
$$

se tiene que

- $\left(\mathrm{D}\left(A^{\alpha}\right),|.|_{\alpha}\right)$ es un espacio de Hilbert.

- Si $\alpha>\beta, D\left(A^{\alpha}\right) \subset D\left(A^{\beta}\right)$ y la inmersión de $\mathrm{D}\left(\mathrm{A}^{\alpha}\right)$ en $\subset D\left(A^{\beta}\right)$ es compacta.

La función (no - lineal) $M$ satisface las siguientes condiciones:

H-1 $M \in \mathrm{C}^{1}([0, \infty)), M(s) \geq-\sigma, \quad \sigma \in \mathbf{R}$. 
Teorema 1. Sean $\alpha, \beta, \varepsilon$ números reales no-negativos tales que:

1. $0 \leq 4 \alpha<2 \beta \leq \varepsilon$.

2. $u_{0} \in D\left(A^{\varepsilon / 2}\right)$

3. $u_{1} \in H$

4. $f \in L^{2}(0, T ; H)$

Entonces existe una función vectorial $u:[0, \mathrm{~T}] \rightarrow D\left(A^{\alpha+(\varepsilon-\beta) / 2}\right)$, tal que :

5. $u \in L^{\infty}\left(0, T ; D\left(A^{\varepsilon / 2}\right)\right)$

6. $u^{\prime} \in L^{\infty}(0, T ; H)$

7. $u^{\prime \prime} \in L^{\infty}\left(0, T ; D\left(A^{\alpha-(\varepsilon-\beta) / 2}\right)\right)$

8. $\frac{d}{d t}\left(u^{\prime}(t), v\right)+\left(A^{\varepsilon / 2} u(t), A^{\varepsilon / 2} v\right)+M\left(\left|A^{\alpha} u(t)\right|^{2}\right)\left(A^{\beta / 2} u(t), A^{\beta / 2} v\right)=(f(t), v)$ $\forall v \in L^{2}\left(0, T ; D\left(A^{\beta / 2}\right)\right)$.

Demostración. Sea $V_{m}=\left[w_{1}, \ldots \ldots w_{m}\right]$, el subespacio generado por los primeros $\mathrm{m}$ vectores propios del operador $A$. Luego $V_{m}$ es un subespacio de $V$ de dimensión finita $\mathrm{m}$, e invariante bajo la acción del operador $A^{\delta}, \forall \delta \in \mathbf{R}$, es decir $A^{\delta}\left(V_{m}\right) \subset V_{m}$.

Sea tambien:

$$
u_{m}(t)=\sum_{i=1}^{m} g_{i m}(t) w_{i} \in V_{m}
$$

donde las funciones $g_{i m}$, son determinadas por la solución del siguiente sistema de ecuaciones diferenciales ordinarias no - lineales :

$$
\begin{aligned}
& \left(u_{m}^{\prime \prime}(t), w_{j}\right)+\left(A^{\varepsilon} u_{m}(t), w_{j}\right)+M\left(\left|A^{\alpha} u_{m}(t)\right|^{2}\right)\left(A^{\beta} u_{m}(t), w_{j}\right)=\left(f(t), w_{j}\right) \\
& j=1,2, \cdots, m \\
& u_{m}(0)=u_{0 m} \rightarrow u_{0} \quad \text { en } \quad D\left(A^{\varepsilon / 2}\right) \\
& u_{m}^{\prime}(0)=u_{1 m} \rightarrow u_{1} \quad \text { en } \quad H
\end{aligned}
$$


Este sistema, luego de un análisis y aplicación del Teorema de Caratheodory sobre existencia local de solución de ecuaciones diferenciales ordinarias no-lineales, admite solución en un intervalo $\left[0, t_{m}\right)$, de donde seguimos la existencia de las soluciones aproximadas $u_{m}$ para $\mathrm{m} \geq 1$. Seguidamente debemos obtener estimados a priori, para la sucesión $\left\{u_{m}\right\}$ de modo que podamos prolongarlas a un intervalo uniforme de existencia .

Estimado a Priori 1. Es fácil ver que la ecuación (9) se verifica para todo $v \in V_{m}$. Entonces reemplazando $w_{j}$ por $v=2 A^{2 \alpha-\beta} u_{m}^{\prime}(t) \in V_{m}$, obtenemos:

$$
\begin{gathered}
\frac{d}{d t}\left\{\left|A^{\alpha-\beta / 2} u_{m}^{\prime}(t)\right|^{2}+\left|A^{\alpha+(\varepsilon-\beta) / 2} u_{m}(t)\right|^{2}+\hat{M}\left(\left|A^{\alpha} u_{m}(t)\right|^{2}\right)\right\} \\
=\frac{d}{d t}\left\{\left|A^{\alpha-\beta / 2} u_{m}^{\prime}(t)\right|^{2}+\left|A^{\alpha+\varepsilon / 2-\beta / 2} u_{m}(t)\right|^{2}+\hat{M}\left(\left|A^{\alpha} u_{m}(t)\right|^{2}\right)+\sigma\left|A^{\alpha} u_{m}(t)\right|^{2}\right\} \\
=\left(f(t), 2 A^{2 \alpha-\beta} u_{m}^{\prime}(t)\right)+2 \sigma\left(A^{\alpha} u_{m}(t), A^{\alpha} u_{m}^{\prime}(t)\right) \\
=2\left(A^{\alpha-\beta / 2} f(t), A^{\alpha-\beta / 2} u_{m}^{\prime}(t)\right)+2 \sigma\left(A^{\alpha+\beta / 2} u_{m}(t), A^{\alpha-\beta / 2} u_{m}^{\prime}(t)\right) \\
\leq\left|A^{\alpha-\beta / 2} f(t)\right|^{2}+2\left|A^{\alpha-\beta / 2} u_{m}^{\prime}\right|^{2}+\sigma^{2}\left|A^{\alpha+\beta / 2} u_{m}\right|^{2} \\
\leq\left|A^{\alpha-\beta / 2} f(t)\right|^{2}+2\left|A^{\alpha-\beta / 2} u_{m}^{\prime}\right|^{2}+\sigma^{2} C\left|A^{\alpha+(\varepsilon-\beta) / 2} u_{m}\right|^{2}
\end{gathered}
$$

Entonces integrando en la desigualdad (13), sobre $[0, t)$ :

$$
\begin{aligned}
& \left|A^{\alpha-\beta / 2} u_{m}^{\prime}(t)\right|^{2}+\left|A^{\alpha+(\varepsilon-\beta) / 2} u_{m}(t)\right|^{2} \\
& \leq\left|A^{\alpha-\beta / 2} u_{m}^{\prime}(0)\right|^{2}+\left|A^{\alpha+(\varepsilon-\beta) / 2} u_{m}(t)\right|^{2}+M\left(\left|A^{\alpha} u_{m}(0)\right|^{2}\right)+\sigma\left|A^{\alpha} u_{m}(0)\right|^{2} \\
& \leq\left|A^{\alpha-\beta / 2} u_{m}^{\prime}(0)\right|^{2}+\left|A^{\alpha+(\varepsilon-\beta) / 2} u_{m}(0)\right|^{2}+\int\left|A^{\alpha-\beta / 2} f(s)\right|^{2} d s+ \\
& +2 \int_{0}^{1}\left|A^{\alpha-\beta / 2} u_{m}^{\prime}(s)\right|^{2} d s+\sigma^{2} C \int_{0}^{1}\left|A^{\alpha+(\varepsilon-\beta) / 2} u_{m}(s)\right|^{2} d s \\
& \leq\left|A^{\alpha-\beta / 2} u_{1 m}\right|^{2}+\left|A^{\alpha+(\varepsilon-\beta) / 2} u_{0 m}\right|^{2}+M\left(\left|A^{\alpha} u_{0 m}\right|^{2}\right)+\sigma \mid A^{\alpha} u_{m}(0)^{2}+ \\
& +\int_{0}^{1}\left|A^{\alpha-\beta / 2} f(s)\right|^{2} d s+2 \int_{0}\left|A^{\alpha-\beta / 2} u_{m}^{\prime}(s)\right|^{2} d s+\sigma^{2} C \int_{0}\left|A^{\alpha+(\varepsilon-\beta) / 2} u_{m}(s)\right|^{2} d s \\
& \leq C+C\left\{\int_{0}^{\prime}\left|A^{\alpha-\beta / 2} u_{m}^{\prime}(s)\right|^{2} d s+\int_{0}^{t}\left|A^{\alpha+(\varepsilon-\beta) / 2} u_{m}(s)\right|^{2} d s\right\}
\end{aligned}
$$

Utilizando (1), las convergencias (10), (11) y la hipótesis $H$-1 sobre $M$, obtenemos una desigualdad de la forma:

$$
\varphi(t)=\left|A^{\alpha-\beta / 2} u_{m}^{\prime}(t)\right|+\left|A^{\alpha+(\varepsilon-\beta) / 2} u_{m}(t)\right|^{2} \leq C+C \int_{0}^{t} \varphi(s) d s
$$


En efecto:

$$
\begin{aligned}
& u_{0 m} \rightarrow u_{0} \text { en } D\left(A^{\varepsilon / 2}\right) \subset D\left(A^{\alpha+(\varepsilon-\beta) / 2}\right) \subset D\left(A^{\alpha}\right) ; \text { luego } \\
& \left|A^{\alpha} u_{0 m}\right|^{2} \rightarrow\left|A^{\alpha} u_{0}\right|^{2} \text { en } R \\
& \left|A^{\alpha+(\varepsilon-\beta) / 2} u_{0 m}\right|^{2} \rightarrow\left|A^{\alpha+(\varepsilon-\beta) / 2} u_{0}\right|^{2} \quad \text { en } R
\end{aligned}
$$

Entonces por la continuidad de M:

$$
M\left(\left|A^{\alpha} u_{0 m}\right|\right) \rightarrow M\left(\left|A^{\alpha} u_{0}\right|\right) \text { en } R
$$

Análogamente

$$
\begin{aligned}
& u_{t m} \rightarrow u_{1} \text { en } H \subset D\left(A^{\alpha-\beta / 2}\right) ; \text { entonces: } \\
& \left|A^{\alpha-\beta / 2} u_{1 m}\right|^{2} \rightarrow\left|A^{\alpha-\beta / 2} u_{1}\right|^{2} \text { en } R
\end{aligned}
$$

Entonces aplicando el lema de Gronwall a (15), obtenemos que:

$$
\left|A^{\alpha+\beta / 2} u_{m}^{\prime}(t)\right|^{2}+\left|A^{\alpha+(\varepsilon-\beta) / 2} u_{m}(t)\right|^{2} \leq C
$$

Estimado a Priori 2. Haciendo $v=2 u_{m}^{\prime}(t)$ en la ecuación aproximada (9), obtenemos:

$$
\begin{aligned}
& \frac{d}{d t}\left\{\left|u_{m}^{\prime}(t)\right|^{2}+\left|A^{\varepsilon / 2} u_{m}(t)\right|^{2}+\Psi(t)\left|A^{\beta / 2} u_{m}(t)\right|^{2}\right\}= \\
& =\frac{d}{d t}\left\{\left|u_{m}^{\prime}(t)\right|^{2}+\left|A^{\varepsilon / 2} u_{m}(t)\right|^{2}+(\Psi(t)+\sigma)\left|A^{\beta / 2} u_{m}(t)\right|^{2}\right\}= \\
& =2\left(f(t), u_{m}^{\prime}(t)\right)+2 \sigma\left(A^{\beta / 2} u_{m}(t), A^{\beta / 2} u_{m}^{\prime}(t)\right)+\Psi^{\prime}(t)\left|A^{\beta / 2} u_{m}(t)\right|^{2}= \\
& =2\left(f(t), u_{m}^{\prime}(t)\right)+2 \sigma\left(A^{\beta} u_{m}(t), u_{m}^{\prime}(t)\right)+\Psi^{\prime}(t)\left|A^{\beta / 2} u_{m}(t)\right|^{2}
\end{aligned}
$$

donde

$$
\begin{aligned}
& \Psi(t)=M\left(\left|A^{\alpha} u_{m}(t)\right|^{2}\right) \\
& \begin{aligned}
\Psi(t) & =2 M^{\prime}\left(\left|A^{\alpha} u_{m}(t)\right|^{2}\right)\left(A^{\alpha} u_{m}(t), A^{\alpha} u_{m}^{\prime}(t)\right) \\
& =2 M^{\prime}\left(\left|A^{\alpha} u_{m}(t)\right|^{2}\right)\left(A^{\alpha+\beta / 2} u_{m}(t), A^{\alpha-\beta / 2} u_{m}^{\prime}(t)\right.
\end{aligned}
\end{aligned}
$$


Ahora teniendo en cuenta la acotación (16), la continuidad de la función $M$, y la condición $0<2 \beta \leq \varepsilon$ (que implica: $\alpha+(\varepsilon-\beta) / 2 \geq \alpha+\beta / 2 \geq \beta / 2$ ):

$$
\begin{aligned}
\left|\Psi^{\prime}(t)\right|=\left|2 M^{\prime}\left(\left|A^{\alpha} u_{m}(t)\right|^{2}\right)\left(A^{\alpha+\beta / 2} u_{m}(t), A^{\alpha-\beta / 2} u_{m}^{\prime}(t)\right)\right| \leq \\
\leq C\left|A^{\alpha+\beta / 2} u_{m}(t)\right|\left|A^{\alpha-\beta / 2} u_{m}^{\prime}(t) \leq\right| \\
\leq C\left|A^{\alpha+(\varepsilon-\beta) / 2} u_{m}(t)\right|\left|A^{\alpha-\beta / 2} u_{m}^{\prime}(t)\right| \leq C
\end{aligned}
$$

Integrando en (17), utilizando la desigualdad de Cauchy - Scharwz , (20), y la hipótesis H-1, obtenemos:

$$
\begin{aligned}
\phi(t)=\left|u_{m}^{\prime}(t)\right|^{2} & +\left|A^{\varepsilon / 2} u_{m}(t)\right|^{2} \leq\left|u_{m}^{\prime}(t)\right|^{2}+\left|A^{\varepsilon / 2} u_{m}(t)\right|^{2}+\Psi(t)+\sigma\left|A^{\beta / 2} u_{m}(t)\right|^{2} \leq \\
\leq & \left|u_{m}^{\prime}(0)\right|^{2}+\left|A^{\varepsilon / 2} u_{m}(0)\right|^{2}+(\Psi(0)+\beta)\left|A^{\beta / 2} u_{m}(0)\right|^{2}+ \\
& +\int_{0}^{t}|f(s)|^{2} d s+\int_{0}^{t}\left|u_{m}^{\prime}(s)\right|^{2} d s+\sigma^{2} \int_{0}^{t}\left|A^{\beta} u_{m}(s)\right|^{2} d s+C \int_{0}^{t}\left|A^{\beta / 2} u_{m}(s)\right|^{2} d s \\
& C \int_{0}^{t}\left|u_{m}^{\prime}(s)\right|^{2} d s+C \int_{0}^{t}\left|A^{\varepsilon / 2} u_{m}(s)\right|^{2} d s \leq C+C \int_{0}^{t} \phi(s) d s
\end{aligned}
$$

Aplicando el lema de Gronwall en (21) obtenemos la acotación:

$$
\phi(t)=\left|u_{m}^{\prime}(t)\right|^{2}+\left|A^{\varepsilon / 2} u_{m}(t)\right|^{2} \leq C \quad \forall t \in[0, T]
$$

\section{Convergencia de las Soluciones Aproximadas}

De (16) y (22) tenemos que:

$$
\begin{aligned}
& \left(u_{m}\right) \text { es acotada en } L^{\infty}\left(0, T ; D\left(A^{\varepsilon / 2}\right)\right) \subset L^{\infty}\left(0, T ; D\left(A^{\alpha}\right)\right) \\
& \left(u_{m}^{\prime}\right) \text { es acotada en } L^{\infty}(0, T ; H)
\end{aligned}
$$

Entonces existe una subsucesión de $\left(u_{m}\right)$ que la seguimos denotando de la misma forma, y una función $u$ tal que:

$$
\begin{aligned}
& u_{m} \stackrel{m \rightarrow \infty}{\longrightarrow} u \text { débil - * en } L^{\infty}\left(0, T_{0} ; D\left(A^{\varepsilon / 2}\right)\right) \\
& u_{m}^{\prime} \stackrel{m \rightarrow \infty}{\longrightarrow} u \text { débil - * en } L^{\infty}\left(0, T_{0} ; H\right)
\end{aligned}
$$


Desde (25) y (26), podemos pasar al limite en los términos lineales de la ecuación aproximada, desde que:

$$
\begin{aligned}
& \int_{0}^{T_{1}}\left(A^{\varepsilon / 2} u_{m}(t), A^{\varepsilon / 2} \omega(t)\right) d t \stackrel{m \rightarrow \infty}{\longrightarrow} \int_{0}^{T_{1}}\left(A^{\varepsilon / 2} u(t), A^{\varepsilon / 2} \omega(t)\right) d t \\
& \int_{0}^{T_{1}}\left(u_{m}^{\prime}(t), \omega(t)\right) d t \stackrel{m \rightarrow \infty}{\longrightarrow} \int_{0}^{T_{1}}\left(u^{\prime}(t), \omega(t)\right) d t \\
& \forall \omega \in L^{1}\left(0, T_{0} ; D\left(A^{\varepsilon / 2}\right)\right) .
\end{aligned}
$$

\section{Análisis del Término No Lineal}

La parte no lineal del problema esta dada por el término:

$$
N\left(u_{m}\right)=M\left(\left|A^{\alpha} u_{m}\right|^{2}\right) A^{\beta / 2} u_{m}
$$

Probaremos que:

$$
N\left(u_{m}\right)=M\left(\left|A^{\alpha} u_{m}\right|^{2}\right) A^{\beta / 2} u_{m} \stackrel{m \rightarrow \infty}{\longrightarrow} N(u)=M\left(\left|A^{\alpha} u\right|^{2}\right) A^{\beta / 2} u
$$

débil en $L^{2}(0, T)$

En efecto, de (16) y (22)

$$
\begin{aligned}
& \left(u_{m}\right) \quad \text { es acotada en } L^{\infty}\left(0, T ; D\left(A^{\varepsilon / 2}\right)\right) \\
& \left(u_{m}^{\prime}\right) \quad \text { es acotada en } L^{\infty}(0, T ; H)
\end{aligned}
$$

Haciendo

$$
B_{0}=D\left(A^{\varepsilon / 2}\right) \subset B=D\left(A^{\alpha}\right) \subset B_{1}=H
$$

Entonces aplicando el teorema de Aubin's - Lions (teniendo en cuenta que la inmersión de $B_{0}$ en $B$ es compacta, existe una subsucesión de $\left(u_{m}\right)$, que la continuamos denotando de la misma forma, tal que:

$$
u_{m} \stackrel{m \rightarrow \infty}{\longrightarrow} u \quad \text { fuerte en } L^{4}\left(0, T ; D\left(A^{\alpha}\right)\right)
$$


Luego:

$$
\left|A^{\alpha} u_{m}\right| \stackrel{m \rightarrow \infty}{\longrightarrow}\left|A^{\alpha} u\right| \quad \text { fuerte en } L^{4}(0, T)
$$

de donde:

$$
\left|A^{\alpha} u_{m}\right|^{2} \stackrel{m \rightarrow \infty}{\longrightarrow}\left|A^{\alpha} u\right|^{2} \quad \text { fuerte en } \quad L^{2}(0, T)
$$

Por otro lado tenemos que la función $M$ es derivable, entonces por el teorema del valor intermedio:

$$
M\left(s_{1}\right)-M\left(s_{2}\right)=M^{\prime}(\tau)\left(s_{1}-s_{2}\right)
$$

de donde: $\tau=\theta s_{1}+(1-\theta) s_{2}, 0 \leq \theta \leq 1$.

Ahora haciendo:

$$
s_{1}(t)=\left|A^{\alpha} u_{m}(t)\right|^{2} \quad ; \quad s_{2}(t)=\left|A^{\alpha} u(t)\right|^{2}
$$

Obtenemos la siguiente convergencia en $L^{2}(0, T)$ :

$$
\begin{array}{rl}
\int_{0}^{T_{1}}\left|M\left(\left|A^{\alpha} u_{m}(t)\right|^{2}\right)-M\left(\left|A^{\alpha} u_{m}(t)\right|^{2}\right)\right|^{2} & d t \int_{0}^{T_{1}}\left|M^{\prime}(\tau(t))\right|\left(\left|A^{\alpha} u_{m}(t)\right|^{2}-\left|A^{\alpha} u(t)\right|^{2}\right)^{2} d t \\
& \leq\left. C \int_{0}^{T_{1}}|| A^{\alpha} u_{m}(t)\right|^{2}-\left.\left|A^{\alpha} u(t)\right|^{2}\right|^{2} d t \stackrel{m \rightarrow \infty}{\longrightarrow} 0
\end{array}
$$

Sea entonces $w=v \theta \in \mathrm{L}^{1}\left(0, T_{0} ; D\left(A^{\beta / 2}\right)\right)$, donde $v \in D\left(A^{\beta / 2}\right)$ y $\theta \in C^{1}\left(\left[0, T_{0}\right]\right)$

$$
\begin{aligned}
\int_{0}^{T_{1}}\left(M\left(\left|A^{\alpha} u_{m}(t)\right|^{2}\right)\right. & \left.\left(A^{\beta / 2} u_{m}(t), A^{\beta / 2}\right)-M\left(\left|A^{\alpha} u(t)\right|^{2}\right)\left(A^{\beta / 2} u(t), A^{\beta / 2} v\right)\right) \theta(t) d t= \\
= & \int_{0}^{T_{1}}\left(M\left(\left|A^{\alpha} u_{m}(t)\right|^{2}\right)-M\left(\mid A^{\alpha} u_{m}(t)^{2}\right)\left(A^{\beta / 2} u_{m}(t), A^{\beta / 2} v\right)\right) \theta(t) d t+ \\
& +\int_{0}^{T_{1}}\left(M\left(\left|A^{\alpha} u(t)\right|^{2}\right)\left(A^{\beta / 2} u_{m}(t)-A^{\beta / 2} u(t), A^{\beta / 2} v\right)\right) \theta(t) d t
\end{aligned}
$$


Por (22) y (39) tenemos que:

$$
\begin{aligned}
& \left|\int_{0}^{T_{1}}\left(M\left(\left|A^{\alpha} u_{m}(t)\right|^{2}\right)-M\left(\left|A^{\alpha} u(t)\right|^{2}\right)\left(A^{\beta / 2} u_{m}(t), A^{\beta / 2} v\right)\right) \theta(t) d t\right| \\
& \quad \leq \int_{0}^{T_{1}}\left|M\left(\left|A^{\alpha} u_{m}(t)\right|^{2}\right)-M\left(\left|A^{\alpha} u(t)\right|^{2}\right)\right| A^{\alpha} u_{m}(t)|| A^{\beta / 2} v|| \theta(t) \mid d t \\
& \quad \leq C \int_{0}^{T_{1}}\left|M\left(\left|A^{\alpha} u_{m}(t)\right|^{2}\right)-M\left(\left|A^{\alpha} u(t)\right|^{2}\right)\right| d t \stackrel{m \rightarrow \infty}{\longrightarrow} 0 .
\end{aligned}
$$

Por otro lado, desde (25) obtenemos:

$$
\begin{aligned}
& \left|\int_{0}^{T}\left(M\left(\left|A^{\alpha} u(t)\right|^{2}\right)\left(A^{\beta / 2} u_{m}(t)-A^{\beta / 2} u(t), A^{\beta / 2} v\right)\right) \theta(t) d\right| \\
& \quad \leq \int_{0}^{T}\left|M\left(\left|A^{\alpha} u(t)\right|^{2}\right)\right|\left|\left(A^{\beta / 2} u_{m}(t)-A^{\beta / 2} u(t), A^{\beta / 2} v \cdot \theta(t)\right)\right| d t \\
& \quad \leq C \int_{0}^{T}\left|\left(A^{\beta / 2} u_{m}(t)-A^{\beta / 2} u(t), A^{\beta / 2} v \cdot \theta(t)\right)\right| d t \stackrel{m \rightarrow \infty}{\longrightarrow} 0
\end{aligned}
$$

Ahora pasaremos al limite en la ecuación aproximada. Desde (10) tenemos:

$$
\begin{aligned}
& \left(u_{m}^{\prime \prime}(t), v\right)+\left(A^{\varepsilon / 2} u_{m}(t), A^{\varepsilon / 2} v\right)+M\left(\left|A^{\alpha} u_{m}(t)\right|^{2}\right)\left(A^{\beta / 2} u_{m}(t), A^{\beta / 2} v\right)=(f(t), v) \\
& \forall v \in V_{m}
\end{aligned}
$$

Multiplicando por $\theta \in D(0, T)$ en (43) e integrando sobre el intervalo [ $0, T]$ obtenemos

$$
\begin{aligned}
\int_{0}^{T}\left(u_{m}^{\prime}(t), v\right) \theta^{\prime}(t) d t & +\int_{0}^{T}\left(A^{\varepsilon / 2} u_{m}(t), A^{\varepsilon / 2} v\right) \theta(t) d t+ \\
& +\int_{0}^{T} M\left(\left|A^{\alpha} u_{m}(t)\right|^{2}\right)\left(A^{\beta / 2} u_{m}(t), A^{\beta / 2} v\right) \theta(t) d t=\int_{0}^{T}(f(t), v) \theta(t) d t
\end{aligned}
$$

$\forall v \in V_{m}$ 
Entonces pasando al limite $\mathrm{m} \rightarrow \infty$ en (44) y por las convergencias (27), (28) y (30)

$$
\begin{array}{r}
\int_{0}^{T}\left(u^{\prime}(t), v\right) \theta^{\prime}(t) d t+\int_{0}^{T}\left(A^{\varepsilon / 2} u(t), A^{\varepsilon / 2} v\right) \theta(t) d t+\int_{0}^{T} M\left(\left|A^{\alpha} u(t)\right|^{2}\right)\left(A^{\beta / 2} u(t), A^{\beta / 2} v\right) \theta(t) d t= \\
=\int_{0}^{T}(f(t), v) \theta(t) d t
\end{array}
$$

$\forall v \in V_{m}$

Por argumentos de densidad, se verifica que

$$
\begin{aligned}
\int_{0}^{T}\left(u^{\prime}(t), v\right) \theta^{\prime}(t) d t+\int_{0}^{T}\left(A^{\varepsilon / 2} u(t), A^{\varepsilon / 2} v\right) \theta(t) d t+\int_{0}^{T} M\left(\left|A^{\alpha} u(t)\right|^{2}\right)\left(A^{\beta / 2} u(t), A^{\beta / 2} v\right) \theta(t) d t= \\
=\int_{0}^{T}(f(t), v) \theta(t) d t \\
\forall v \in D\left(A^{\beta / 2}\right)
\end{aligned}
$$

Luego:

$$
\frac{d}{d t}\left(u^{\prime}(t), v\right)+\left(A^{\varepsilon / 2} u(t), A^{\varepsilon / 2} v\right)+M\left(\left|A^{\alpha} u(t)\right|^{2}\right)\left(A^{\beta / 2} u(t), A^{\beta / 2} v\right)=(f(t), v)
$$

en el sentido de las distribuciones sobre $\left[0, T_{0}\right]$ y para todo $v \in D\left(A^{\beta / 2}\right)$

$$
u^{\prime \prime}=-M\left(\left|A^{\alpha} u(t)\right|^{2}\right) A^{\beta} u-A^{\varepsilon} u+f \in L^{2}\left(0, T ; D\left(A^{-\varepsilon / 2}\right)\right)
$$

\section{Verificación de las Condiciones Iniciales}

Tenemos por (3), (40) y (41) que:

$$
\begin{aligned}
& u \in L^{\infty}\left(0, T ; D\left(A^{\varepsilon / 2}\right)\right), \text { entonces: } \\
& A^{\beta} u \in L^{\infty}\left(0, T ; D\left(A^{\varepsilon / 2-\beta}\right)\right) \subset L^{\infty}\left(0, T ; D\left(A^{-\varepsilon / 2}\right)\right) \\
& A^{\varepsilon} u \in L^{\infty}\left(0, T ; D\left(A^{-\varepsilon / 2}\right)\right) \\
& f \in L^{2}(0, T ; H) \subset L^{2}\left(0, T ; D\left(A^{-\varepsilon / 2}\right)\right)
\end{aligned}
$$

Por lo tanto:

$$
\begin{aligned}
& u \in C_{w}^{0}\left([0, T] ; D\left(A^{\varepsilon / 2}\right)\right) \\
& u^{\prime} \in C_{w}^{0}([0, T] ; H)
\end{aligned}
$$


entonces

$$
\begin{aligned}
& u(0) \in D\left(A^{\varepsilon / 2}\right) \subset H \\
& u^{\prime}(0) \in H
\end{aligned}
$$

Sea $\theta \in C^{1}([0, T]), \operatorname{con} \theta(T)=0$ y $\theta(0)=1$. Entonces:

$$
\begin{aligned}
& \int_{0}^{T}\left(u_{m}^{\prime}(t), v\right) \theta(t) d t=\int_{0}^{T} \frac{d}{d t}\left(u_{m}(t), v\right) \theta(t) d t=\left(u_{m}(0), v\right)-\int_{0}^{T}\left(u_{m}(t), v\right) \theta^{\prime}(t) d t \\
& =\left(u_{0 m}, v\right)-\int_{0}^{T}\left(u_{m}(t), v\right) \theta^{\prime}(t) d t \rightarrow\left(u_{0}, v\right)-\int_{0}^{T}(u(t), v) \theta^{\prime}(t) d t
\end{aligned}
$$

Por otro lado

$$
\begin{aligned}
& \int_{0}^{T}\left(u_{m}^{\prime}(t), v\right) \theta(t) d t=\int_{0}^{T} \frac{d}{d t}\left(u_{m}(t), v\right) \theta(t) d t \rightarrow \int_{0}^{T} \frac{d}{d t}(u(t), v) \theta(t) d t \\
& \quad=(u(0), v)-\int_{0}^{T}(u(t), v) \theta^{\prime}(t) d t
\end{aligned}
$$

Comparando (53) y (54)

$$
(u(0), v)=\left(u_{0}, v\right) \quad \forall v \in D\left(A^{\varepsilon / 2}\right)
$$

y desde que $D\left(A^{\varepsilon / 2}\right)$ es denso en $H$

$$
u(0)=u_{0}
$$

Probaremos que $u^{\prime}(0)=u_{1}$

Sea $\delta>0 \quad y$

$k_{\delta}= \begin{cases}1-t / \delta & 0 \leq t \leq \delta \\ 0 & \delta<t \leq T\end{cases}$

multiplicando ambos lados de la ecuación aproximada, por $\kappa_{\delta}$, e integrando de 0 a $T$,

$$
\begin{gathered}
\int_{0}^{T}\left(\left(u_{m}^{\prime \prime}(t), v\right)+\left(A^{\varepsilon / 2} u_{m}(t), A^{\varepsilon / 2} v\right)+M\left(\left|A^{\alpha} u_{m}(t)\right|^{2}\right)\left(A^{\beta / 2} u_{m}(t), A^{\beta / 2} v\right)\right) k_{\delta}(t) d t= \\
=\int_{0}^{T}(f(t), v) k_{\delta} d t \\
\forall v \in D\left(A^{\varepsilon / 2}\right)
\end{gathered}
$$


entonces tenemos

$$
\begin{aligned}
& \int_{0}^{T}\left(u_{m}^{\prime \prime}(t), v\right) k_{\delta}(t) d t=\frac{d}{d t}\left(u_{m}^{\prime}(t), v\right) k_{\delta}(t) d t=\left(u_{m}^{\prime}(0), v\right)+\int_{0}^{\delta}\left(u_{m}^{\prime}(t), v\right) k_{\delta}^{\prime}(t) d t \\
& =-\left(u_{1 m}, v\right)+\frac{1}{\delta} \int_{0}^{\delta}\left(u_{m}^{\prime}(t), v\right) d t \rightarrow\left(u_{1}, v\right)+\frac{1}{\delta} \int_{0}^{\delta}\left(u^{\prime}(t), v\right) d t \\
& =-\int_{0}^{T} M\left(\left|A^{\alpha} u(t)\right|^{2}\right)\left(A^{\gamma / 2} u(t), A^{\gamma / 2} v\right) k_{\delta}(t) d t \int_{0}^{T}\left(A^{\varepsilon / 2} u(t), v\right) k_{\delta}(t) d t+(f(t), v) k_{\delta}(t) d t \\
& \forall v \in D\left(A^{\varepsilon / 2}\right)
\end{aligned}
$$

Desde que:

$$
\frac{1}{\delta} \int_{0}^{\delta}\left(u^{\prime}(t), v\right) d t \stackrel{\delta \rightarrow 0}{\longrightarrow}\left(u^{\prime}(0), v\right)
$$

$$
\begin{aligned}
& \int_{0}^{T_{1}} M\left(\left|A^{\alpha} u(t)\right|^{2}\right)\left(A^{\beta / 2} u(t), A^{\beta / 2} v\right) k_{\delta}(t) d t=\int_{0}^{\delta} M\left(\left|A^{\alpha} u(t)\right|^{2}\right)\left(A^{\beta / 2} u(t), A^{\beta / 2} v\right)(1-t / \delta) d t= \\
& =\int_{0}^{\delta} M\left(\left|A^{\alpha} u(t)\right|^{2}\right)\left(A^{\beta / 2} u(t), A^{\beta / 2} v\right) d t-\frac{1}{\delta} \int_{0}^{\delta} M\left(\left|A^{\alpha} u(t)\right|^{2}\right)\left(A^{\beta / 2} u(t), A^{\beta / 2} v\right) t d t \stackrel{\delta \rightarrow 0}{\longrightarrow} 0 \\
& \forall v \in D\left(A^{\beta / 2}\right)
\end{aligned}
$$

obtenemos que $\left(u_{1}, v\right)=\left(u^{\prime}(0), v\right), \forall v \in D\left(A^{\beta / 2}\right)$ y por argumentos de densidad, concluimos que:

$$
u(0)=u_{1}
$$

Observación 1. La unicidad de la solución se sigue fácilmente por la condición impuesta sobre la función no-lineal $M$.

Observación 2. Si los exponentes $\alpha, \beta$, cumplen la condición $2 \alpha-\beta \geq 0$, no es necesario realizar el estimado 2, para las soluciones aproximadas. En este caso el estimado 1, nos dice que $\left(u_{\mathrm{m}}\right)$ es acotada en $\mathrm{D}\left(\mathrm{A}^{\alpha+(\varepsilon-\beta) / 2}\right) \subset \mathrm{D}\left(\mathrm{A}^{\varepsilon / 2}\right)$ y que $\left(u_{\mathrm{m}}^{\prime}\right)$ es acotada en $\mathrm{D}\left(\mathrm{A}^{\alpha-\beta / 2}\right) \subset \mathrm{H}$ y por tanto podemos continuar como en el teorema 1.

3. Caso Degenerado. En esta sección trataremos el caso en que el operador $A$ es auto-adjunto y no-negativo. En Aassilla M. [2] se utilizo un método para tratar ecuaciones degeneradas considerando una descomposición del espacio $H=\mathrm{N}(A) \oplus \mathrm{R}(A)$ y de la ecuación abstracta sobre cada uno de los espacios componentes. Nosotros procedemos de forma similar y consideramos la siguiente situación: 
(3.1) Sean $V, H$ espacios de Hilbert, la inmersión de $V$ en $H$ densa y compacta.

(3.2) $a: V \times V \rightarrow \mathbf{R}$ una forma bilineal, simétrica, continua y no-negativa, tal que

$$
a(v, v)+\lambda|v|_{H}^{2} \geq c|v|_{V}^{2} \quad \forall v \in V
$$

para algún $\lambda \in \Re$ y $c \succ 0$.

Entonces el operador $A_{\lambda}=A+\lambda I$ determinado por la terna $\left\{V, H, a_{\lambda}(u, v)\right\}$, es auto-adjunto, con dominio $\mathrm{D}\left(A_{\lambda}\right)=\mathrm{D}(A)$ denso en $H$ y el operador inverso $A_{\lambda}{ }^{-1}$ es compacto. Entonces estamos en condiciones de utilizar el siguiente resultado cuya demostración aparece en [2].

Lema 1. Sea $H$ un espacio con producto interno sobre los reales. Sea $A$ un operador autoadjunto, no-negativo con dominio $\mathrm{D}(A)$ y rango $\mathrm{R}(A)$ en $H$. Supongamos que $(I+A)^{-1}$ es un operador compacto. Entonces:

a) $\mathrm{R}(A)$ es cerrado y $H=N(A) \oplus \mathrm{R}(A)$

b) $\left(\left.\mathrm{A}\right|_{\mathrm{R}(\mathrm{A})}\right)^{-1}: \mathrm{R}(A) \rightarrow \mathrm{R}(A)$ es compacto, donde $\left.\mathrm{A}\right|_{\mathrm{R}(\mathrm{A})}$ es la restricción de $A$ al $\mathrm{R}(A)$.

Siguiendo [2], planteamos el siguiente problema sobre $H$.

Hallar $u(t)=u_{l}(t)+u_{2}(t)$ donde $u_{l}(t) \in \mathrm{N}(A)$ y $u_{2}(t) \in \mathrm{D}(A) \cap \mathrm{R}(A)$ tal que

$$
\begin{aligned}
& u_{1}^{\prime}(t)+u_{2}^{\prime \prime}(t)+M\left(\left|A^{\alpha}\left(u_{1}(t)+u_{2}(t)\right)^{2}\right|^{2}\right) A^{\beta} u_{2}(t)+A^{\varepsilon} u_{2}(t)=f_{1}(t)+f_{2}(t) \\
& u(0)=u_{1}(0)+u_{2}(0)=u_{01}+u_{02} \\
& u^{\prime}(0)=u_{1}^{\prime}(0)+u_{2}^{\prime}(0)=u_{11}+u_{12}
\end{aligned}
$$

En el caso $\alpha>0$, la solución de (3.1) equivale a la resolución de los problemas siguientes:

Hallar $u(t)=u_{l}(t)+u_{2}(t)$ donde $u_{l}(t) \in \mathrm{N}(A)$ y $u_{2}(t) \in \mathrm{D}(A) \cap \mathrm{R}(A)$ tal que;

$$
\begin{aligned}
& u_{1}^{\prime}(t)=f_{1}(t) \text { en } N(A) \\
& u_{1}(0)=u_{01}, u_{2}(0)=u_{11}
\end{aligned}
$$

y

$$
\begin{aligned}
& u_{1}^{\prime \prime}(t)+M\left(\left|A^{\alpha} u_{2}(t)\right|^{2}\right) A^{\beta} u_{2}(t)+A^{\varepsilon} u_{2}(t)=f_{2}(t) \text { en } R(A) \\
& u_{1}(0)=u_{02} \\
& u_{2}^{\prime}(0)=u_{12}
\end{aligned}
$$


donde en (3.5) estamos denotando de la misma forma la restricción de A a R(A), y se utiliza que $A^{\delta} u_{l}(t)=0, \forall \delta>0$.

Ahora, (3.4) se resuelve trivialmente. Asimismo desde que $A$ restricto al $\mathrm{R}(\mathrm{A})$ es autoadjunto, positivo y con inversa compacta, (que seguimos denotando de la misma forma)podemos aplicar el teorema 1 de la sección anterior para resolver (3.5) y recuperar el teorema 1 para el caso $\alpha>0$.

4. Aplicaciones. Sea $\Omega \subset \mathbf{R}^{\mathrm{n}}$ un conjunto abierto acotado con frontera regular $\Gamma$.

4.1. Sea $H=L^{2}(\Omega) ; V=H_{0}{ }^{l}(\Omega) \cap H^{2}(\Omega)$, consideremos la siguiente forma bilineal sobre $V$ :

$$
a(u, v)=\int \Delta u \Delta v d x
$$

Entonces el operador asociado a la terna $\{V, H, a(u, v)\}$ esta dado por:

$$
A u=\Delta^{2} u \quad \forall u \in D(A)=H_{0}^{1}(\Omega) \cap H^{4}(\Omega)
$$

y se tiene que:

En estas condiciones el teorema 1 afirma la existencia y unicidad de solución del problema:

$$
\mid \begin{array}{ll}
u^{\prime \prime}+\Delta^{2} u+M\left(|\nabla u|^{2}\right)(-\Delta u)=f & \text { en } Q \\
u=\Delta u=0 & \text { en } \Sigma \\
u(0)=u_{0}, u^{\prime}(0)=u_{1} & \text { en } \Omega
\end{array}
$$

4.2. Sea $H=L^{2}(\Omega) ; V=H^{l}(\Omega)$, consideremos la siguiente forma bilineal sobre $V$ :

$$
a(u, v)=\int \nabla u \nabla v d x
$$

Entonces el operador (no - negativo) asociado a la terna $\{V, H, a(u, v)\}$ esta dado por:

$$
A u=\Delta u \quad \forall u \in D(A)=\left\{u \in H^{2}(\Omega) / \frac{\partial u}{\partial \eta}=0\right\}
$$

y se tiene que:

$$
\begin{array}{ll}
A^{2} u=\Delta^{2} u & \forall u \in D\left(A^{2}\right)=H^{2}(\Omega) \\
A^{1 / 2} u=\nabla u & \forall u \in D\left(A^{1 / 2}\right)=H^{1}(\Omega)
\end{array}
$$


En estas condiciones el teorema 1 afirma la existencia y unicidad de solución del problema:

$$
\mid \begin{array}{ll}
u^{\prime \prime}+\Delta^{2} u+M\left(|\nabla u|^{2}\right)(-\Delta u)=f & \text { en } Q \\
\frac{\partial u}{\partial \eta}=0 & \text { en } \Sigma \\
u(0)=u_{0}, u^{\prime}(0)=u_{1} & \text { en } \Omega
\end{array}
$$

\section{REFERENCIAS BIBLIOGRÁFICAS}

1. Arosio A. - Spagnolo S.: "Global solutions of the Cauchy problem for a non-linear Hyperbolic Equation "Universita di Pisa . Departamento de Matemática. Roma (1982).

2. A Assila M. "On a Quasilinear Wave Equation with Strong Damping" Funkcialaj Ekvaioj 41 -(1998)

3. Cousin A. , Frota C. Larkin N, ,Medeiros L.A. "On the abstract model of Kirchhoff-Carrier Equation" Comm. In Appl. Analysis - 3- (1997).

4. Ebihara Y.: "On the existence of local smooth solutions for same degenerate quasilinear hyperbolic equations". Anais Acad.Bras.Ciencias.vol.57 (1985)

5. Ebihara Y. -Medeiros L. - Milla A.: "Local solutions for a nonlinear degenerate hyperbolic equations". Nonlinear Analysis.Vol.10. (1986).

6. Izaguirre R. - Pereira D.: "Solução global para uma classe de equações hiperbólicas degeneradas" Proceding of $9^{0}$ Congreso Brasileiro de Matematica Aplicada e Computacional-Brasilia-(1986)

7. Izaguirre R. - Veliz - V.: " Existencia y unicidad de solucion local para una clase de ecuaciones abstractas no-lineales tipo Kirchhoff-Carrier" I Seminario Internacional de Ecuaciones Diferenciales Parciales y Aplicaciones - Universidad Ricardo Palma - Peru- (1999)

8. Izaguirre R. Veliz V.: " Solución Local para una clase de ecuaciones tipo Kirchhoff" Actas del $42^{0}$ Seminario Brasileiro de analisis (1997).

9. Limaco J. - Ferrel L.: "Existencia de soluções para a equação da corda elástica com amortecimento" Atas do 37 Seminario Brasileiro de Analise

10. Lions J. L.: "Quelques Methodes de Resolution des Probleme aux limites nonlinear". Dunod. Paris (1969).

11. Matos M. "Estudo de um modelo abstrato para a equação da viga vía integral Hilbertiana" Atas $29^{\circ}$ Seminario Brasileiro de Analise- (1989).

12. Medeiros L.A - Milla M.M.: "Remarks on a nonlinear model vibrations of string with damping".30 Seminario Brasileiro de Analise.L.N.C.C.-R.J.(1989)

13. Muñoz J. "Remark on the existence and Decay of the Nonlinear Beam Equation" Internat. J. Math. Vol.17, $\mathrm{N}^{0} 2-($ 1994).

14. Pereira D. C. "Existence, Uniqueness and Asymptotic Behavior for Solutions of the nonlinear Beam equation". Nonlinear Analysis, Theory, Methods and Applications, Vol 14, N0 8, 1990.

15. Perla G.: "On classical solutions of quasilinear hyperbolic equations". Nonlinear Analysis.Vol.3.(1979)

16. Woinowsky S. -Krieger. "The effect of axial force on the vibration of hinged bar" J. Appl. Math. 17 (1950). 